\title{
THE NOBEYAMA MILLIMETER ARRAY
}

\author{
KOH-ICHIRO MORITA \\ Nobeyama Radio Observatory, National Astronomical Observatory, \\ Minamisaku, Nagano 384-13, Japan
}

\begin{abstract}
The present status and the upgrading projects for the Nobeyama Millimeter Array are described. The array is currently being upgraded to produce higher sensitivity and new spectral windows. The major items have been a new $10-\mathrm{m}$ antenna, new receivers, and a new correlator. There follows a brief summary of the astronomical research is presented.
\end{abstract}

\section{INTRODUCTION}

The Nobeyama Millimeter Array (NMA) consists of five 10-m diameter antennas located at Nobeyama $150 \mathrm{~km}$ west of Tokyo at an elevation of $1350 \mathrm{~m}$. The first aperture synthesis observations at $22 \mathrm{GHz}$ were made with the continuum correlator system in 1984 (Ishiguro et al. 1984). We developed an FX type correlator (the Nobeyama FX) for line observations with the maximum bandwidth of 80 $\mathrm{MHz}$ in 1985, which was expanded to $320 \mathrm{MHz}$ in 1987 (Chikada et al. 1987). In 1988, dual frequency SIS receivers covering the $\lambda \sim 7 \mathrm{~mm}$ and $\lambda \sim 2.6 \mathrm{~mm}$ were installed (Kawabe et al. 1990) and spectral line observations at millimeter wavelengths were started(Ishiguro et al. 1990).

We have three major upgrading projects to produce higher sensitivity and new spectral windows: The addition of a new antenna, development of new SIS receivers for the $\lambda \sim 2.6 \mathrm{~mm}, \lambda \sim 2 \mathrm{~mm}$, and $\lambda \sim 1.3 \mathrm{~mm}$, and a new correlator system. The schedule of test operation of the new antenna is in the spring of 1994. The development of the new SIS receivers has been partly completed and the first synthesis images of $\mathrm{CS}(3-2)$ line and $\lambda \sim 2 \mathrm{~mm}$ continuum were obtained in the winter of 1992 (Ishizuki et al. 1993 and Kawabe et al. 1993). Test observations for $\lambda \sim 1.3 \mathrm{~mm}$ will be started in the winter of $1993-1994$. The new correlator system which will be completed in 1994 produces the maximum bandwidth of $2 \mathrm{GHz}$ and will realize better performances for continuum and calibration observations.

\section{ANTENNAS}

The five $10-\mathrm{m}$ diameter antennas have paraboloidal main reflector placed on alt-azimuth mounting. The main reflector consists of 36 aluminium honeycomb panes, the surface accuracies of which are $60 \mu \mathrm{m}$ on an average. Averaged surface accuracy of main dish for five antennas is $71 \mu \mathrm{rms}$.

The antennas are configured for Cassegrain coude optics. Wide band radio 
waves are transmitted to the ground level through the low-loss beam wave-guide system.

The measured nighttime aperture efficiencies at $\lambda=3 \mathrm{~mm}$ and $\lambda=2 \mathrm{~mm}$ are $0.5-0.6$ and $0.25-0.5$ respectively (Fig. 2). These results are consistent with the efficiencies estimated from the surface accuracies of main reflector, subreflector, and the mirrors for the beam wave-guide. The pointing accuracy of the antennas is $\sim 6$ arcsec rms.

The antennas are movable along the two rail tracks which are shown in Fig. 1. There are 30 stations along the tracks: 17 stations along the $560 \mathrm{~m}$ east-west track, 13 stations along the $520 \mathrm{~m}$ north-south track (tilted by 33 degrees from the true north). The astronomical measurements of the baselines are accurate to $<0.3 \mathrm{~mm}$ rms (Morita et al. 1989).

The new antenna realizes good performance at higher frequencies $(>100$ $\mathrm{GHz}$ ), although the basic design (Fig. 3) is same as that of existing antennas.

Surface accuracies of each aluminium panel, the sub-reflector, and the mirrors for beam wave-guide are $\sim 20 \mu \mathrm{m} \mathrm{rms,} \sim 15 \mu \mathrm{m} \mathrm{rms}$, and $\sim 10 \mu \mathrm{m} \mathrm{rms}$ The distribution of achieved panel accuracies is shown in Fig. 4; the average value is $21 \mu \mathrm{m}$ rms. Each panel is attached to the relector support structure at six points. The height of each support point is adjusted by remotely controlled motors, thus allowing a rapid correction of the surface errors. The gravitational deformation in the backup structure is estimated to be $<10 \mu \mathrm{m}$ rms.

The foot position of sub-reflector supports is shifted to the edge of main reflector so that the blocking area due to the sub-reflector supports is minimized.

The optics is designed to be efficient at $100-200 \mathrm{GHz}$. We have adopted a shaping optics for \#3 mirror and \#4 mirror and achieved nearly uniform illumination of the aperture. Theoretical calculation of aperture efficiency (discounting blockage, but including estimated spillover) shows an improvement of $25 \%$ or a gain of $0.84 \mathrm{~dB}$ for aperture efficiency at $115 \mathrm{GHz}$.

As results of these modifications we expect a good performance of the new antenna for observations at sub-millimeter wavelengths.

\section{RECEIVERS}

Each antenna is equipped with a $4 \mathrm{~K}$ cryostat. The cryostats can accomodate three SIS receivers and are presently configured with two SIS mixers; one 80 to $115 \mathrm{GHz}$ mixer and one 140 to $160 \mathrm{GHz}$ mixer. An SIS mixer for $230 \mathrm{GHz}$ is now under development. The refrigeration system is a closed-cycle arrangement consisting of a G-M $15 \mathrm{~K}$ refrigerator and a J-T expansion loop.

The SIS mixers use $\mathrm{Nb} / \mathrm{Al}-\mathrm{AlOx} / \mathrm{Nb}$ junctions developed at Nobeyama (Kawabe et al. 1990). We have adopted the the tunerless mixer mounts (Sunada et al. 1993). Laboratory measurements show that these SIS mixers have low noise temperatures (in DSB) of $<50 \mathrm{~K}$ for a frequency range of 80 to $120 \mathrm{GHz}$ and $\sim 50 \mathrm{~K}$ for 130 to $160 \mathrm{GHz}$ (Fig. 3 in Sunada et al. 1993).

The receivers have been successfully installed in the antennas in the winter of 1991-1992 and the first synthesis images of CS(3-2) line and $\lambda \sim 2 \mathrm{~mm}$ continuum were obtained(Ishizuki et al. 1993 and Suzuki et al. 1993). Installation of the $230 \mathrm{GHz}$ mixer in the antennas is due in the winter of $1993-1994$. 


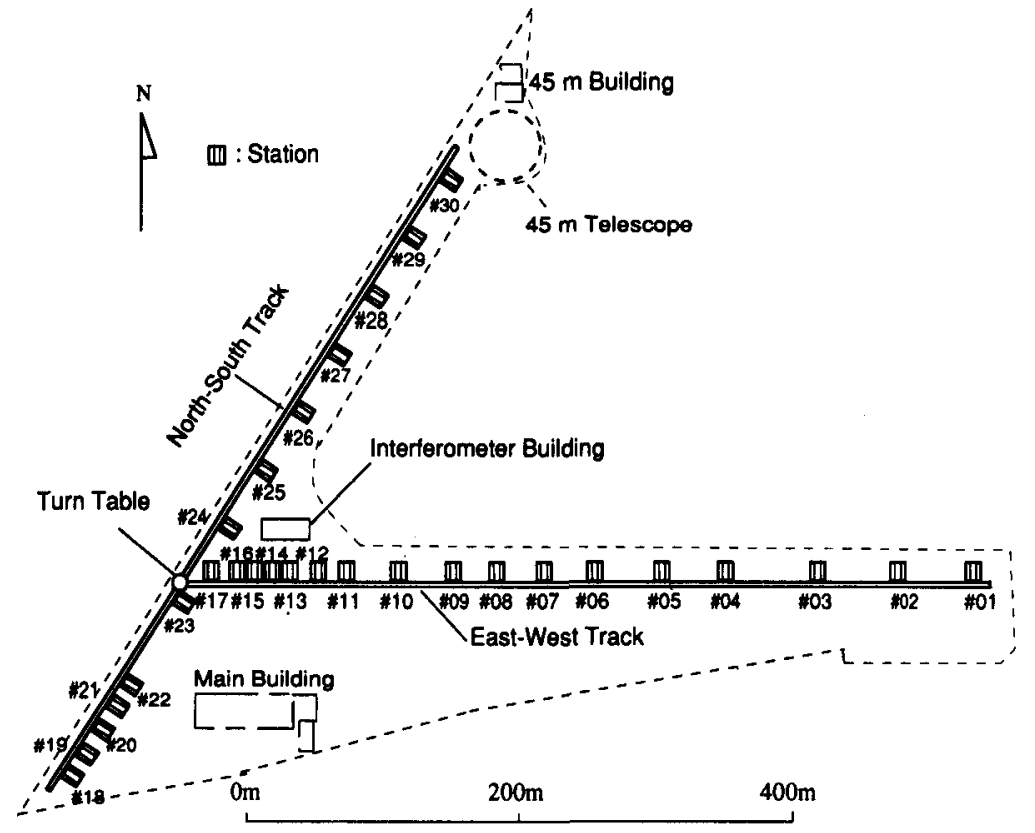

Fig. 1. Track and station layout of the Nobeyama Millimeter Array.
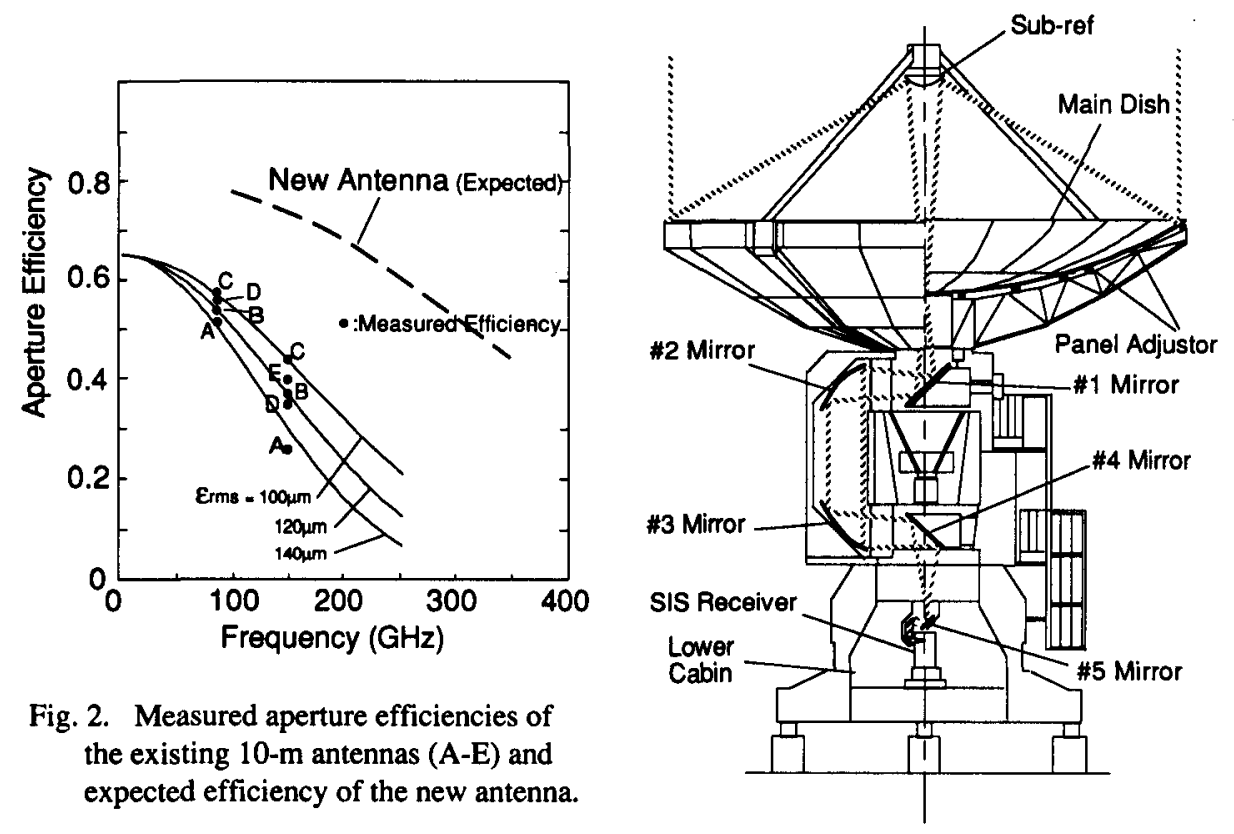

Fig. 3. The basic design of the new 10-m antenna. 


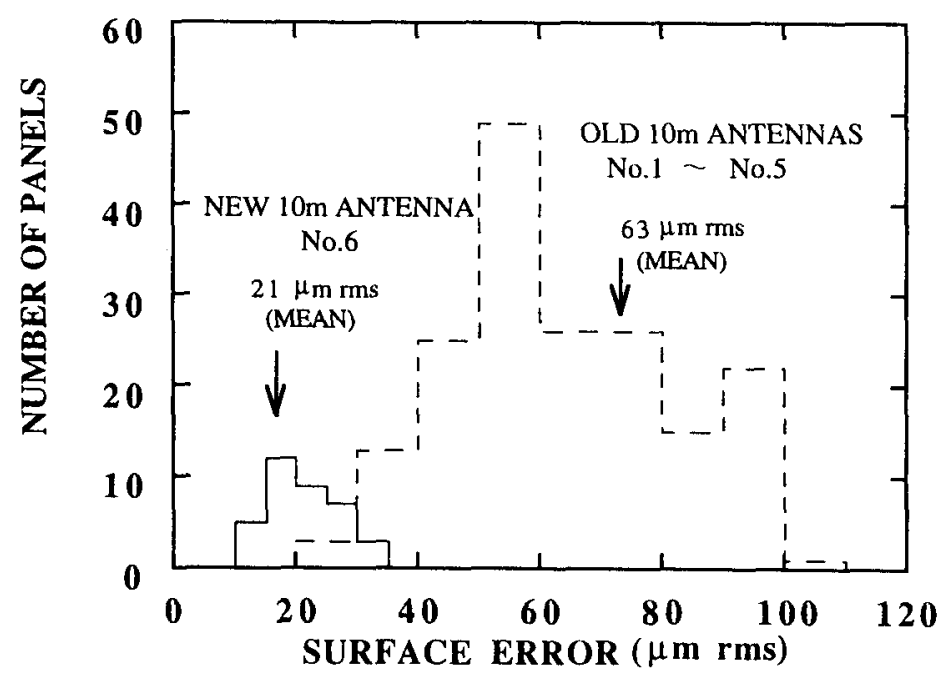

Fig. 4. The distributions of panel accuracies of the existing antennas and the new antenna.

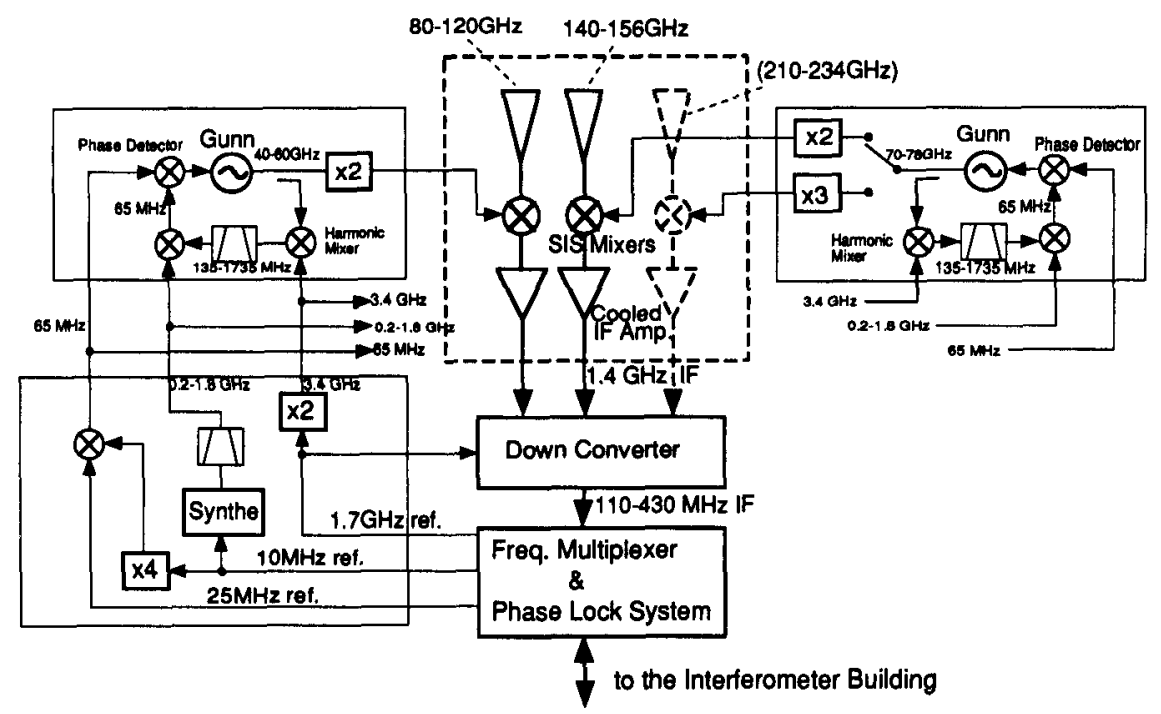

Fig. 5. Block diagram of LO and IF system. 


\section{LO SYSTEM}

The local oscillator of the receiver for each RF band is provided by a GaAs Gunn oscillator and a doubler/a tripler (Fig. 5). The powers of the Gunn oscillators for $\lambda \sim 2.6 \mathrm{~mm}$ and $\lambda \sim 2 \mathrm{~mm}$ are $20 \mathrm{~mW}$ and $20 \sim 30 \mathrm{~mW}$, respectively. We expect to replace the GaAs Gunn oscillator with a high power InP Gunn oscillator in 1993. The oscillators are phase locked to a reference signal in the range of 1686 to $1706 \mathrm{MHz}$.

The reference signal is distributed to each telescope in the array via a phase stable reference system (Ishiguro et al. 1984). A round-trip phase correction system based on the early design of the VLA is used. Fringe rotation and phase switching are applied to the $\mathrm{LO}$ via a $25 \mathrm{MHz}$ reference in the Gunn oscillator phase lock system.

\section{IF SYSTEM}

Each SIS mixer is followed by a cooled FET or HEMT amplifier operating in the 1.2 to $1.6 \mathrm{GHz}$ band with a noise temperature of $5-12 \mathrm{~K}$. The first IF signal is converted to the $110-430 \mathrm{MHz}$ IF signal, which are sent back to the interferometer building via a phase-stabilized coaxial cable. This cable is also used for transmission of the reference signals for the local oscillators.

In order to increase the sensitivity for continuum observations, a new IF system which has a bandwidth of $2 \mathrm{GHz}$ is under development. Frequency range of the new IF signal will be $5-7 \mathrm{GHz}$. New HEMT amplifier and optical fibers will be used for transmission of the wideband IF signals.

\section{CORRELATORS}

For line and continuum observations, the FX type correlator has been operated with a $320 \mathrm{MHz}$ bandwidth $\mathrm{A} / \mathrm{D}$ converter system and digital delays. The A/D converter system uses $320 \mathrm{MHz} 3$-bit samplers. The correlator has 1024 complex frequency channels for each baseline and the highest resolution in frequency is $4.9 \mathrm{kHz}$.

For the new correlator system, we adopt the XF technique, because it is more economical for the NMA and it offers more flexibility. The new correlator system uses a $4 \mathrm{GHz}$ 2-bit sampler and a 32-parallel, $32 \mathrm{MHz}, 32$-lag correlation LSI which can make correlation processing at a rate of $1 \mathrm{GHz}$ (Kawaguchi 1992). The sampler, which has been developed for VLBI observations, has a Si bipolar LSI which contains two flush A/D converters, each of which degitize a signal in 2 bits at every half cycle of a $4 \mathrm{GHz}$ clock. The processing rate of $1 \mathrm{GHz}$ of the correlation LSI is realized by introducing parallel architecture into the LSI design. It is based on a custom VLSI chip (a CMOS gate array) and produces only real cross correlations. With 12 chips of the correlation LSI, the processing on $4 \mathrm{GHz}$, 2-bit samples can be done. 


\section{CONTROL SYSTEM}

The control system of the NMA is a distributed system, based on a Fujitsu S-4/370 workstation (SUN workstation compatible), a Fujitsu M760 mainframe (IBM compatible), and personal computers connected with an ethernet. The mainframe controls the array for accurate tracking and acquires a large amount of data from the Nobeyama FX. Observers can conduct various observing sequences with the workstation by making command tables. It provides flexible GUI based on the X-Window to observers and communicates with the mainframe by using a socket interface. The personal computers control and monitor the receiver system, the LO system, and the IF system.

\section{DATA REDUCTION}

UVPROC, which run on a super computer (Fujitsu VP200E), has been developed for reducing visibility data. Its basic facilities include data display, edting, and calibrations. Observations of reference sources are used to calibrate the bandpass, the flux scale, and the source amplitude and phase. Observers can directly transfer calibrated visibilities to the NRAO AIPS package, which also run on VP200E, or make calibrated FITS uv files. A modified version of UVPROC for UNIX workstations will be available in 1993.

\section{PERFORMANCE}

In order to estimate the imaging performace of the NMA, we have carried out test observations of $3 \mathrm{C} 273$ and $3 \mathrm{C} 84$ at $\lambda \sim 3 \mathrm{~mm}$ (Morita et al. 1989). The synthesis images without "self-calibration" technique showed a dynamic range of 70:1. The absolute positional accuracies were $<10 \%$ of the synthesized beam width. These results are almost consistent with those estimated from the calibration errors and the baseline errors. We have estimated that the relative positional accuracies are $<1 \%$ of the synthesized beam width from the test observations. These positional accuracies were achieved in positional measurements of SiO $J=1-0$ masers in active star-forming region (Morita et al. 1992), which reveal that the $\mathrm{SiO}$ masers in W51 and $\mathrm{Sgr}-\mathrm{B} 2$ coincide with the $\mathrm{H}_{2} \mathrm{O}$ masers and $\mathrm{NH}_{3}$ hot-cores in these sources with $1^{\prime \prime}$.

\section{RECENT OBSERVATIONAL RESULTS}

Various scientific researches have been carried out by the NRO group and the visitors with the NMA. Many astronomical results are appeared in this volume. The followings are highlights of recent results obtained by the NRO group.

Kawabe et al. (1992) have made aperture synthesis CO(3-2) observations of a protogalaxy candidate, IRAS F10214+4724. They have found that molecular gas surface density is as high as that in IR luminous galaxies.

The high resolution $\mathrm{CO}(1-0)$ images of seven IR luminous galaxies have been made. $1.7^{\prime \prime}$ resolution images of ARP220 reveal that a inclined massive gas fing has been formed in the central $1 \mathrm{kpc}$ region (Okumura et al. 1993). 


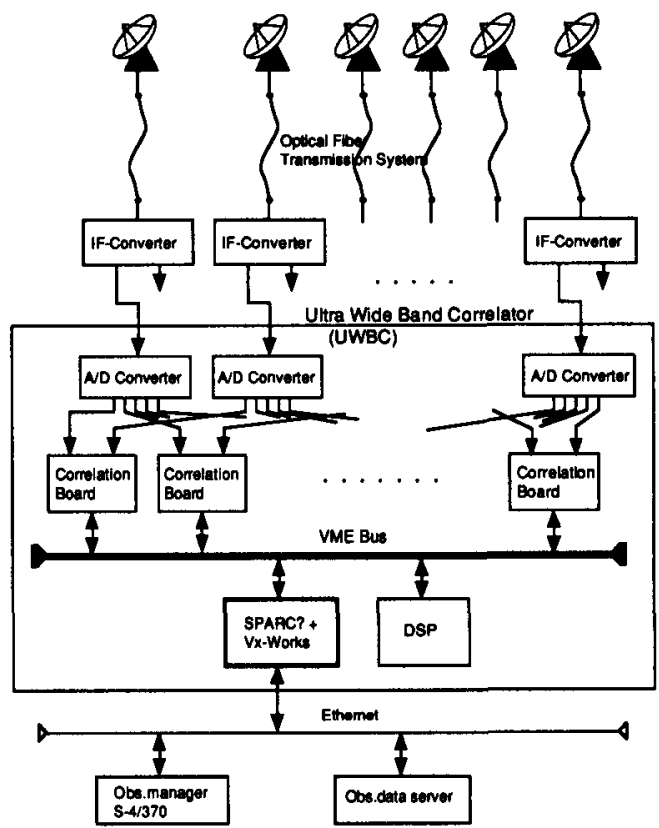

Fig. 6. Ultra Wide Band Correlator (UWBC) system.

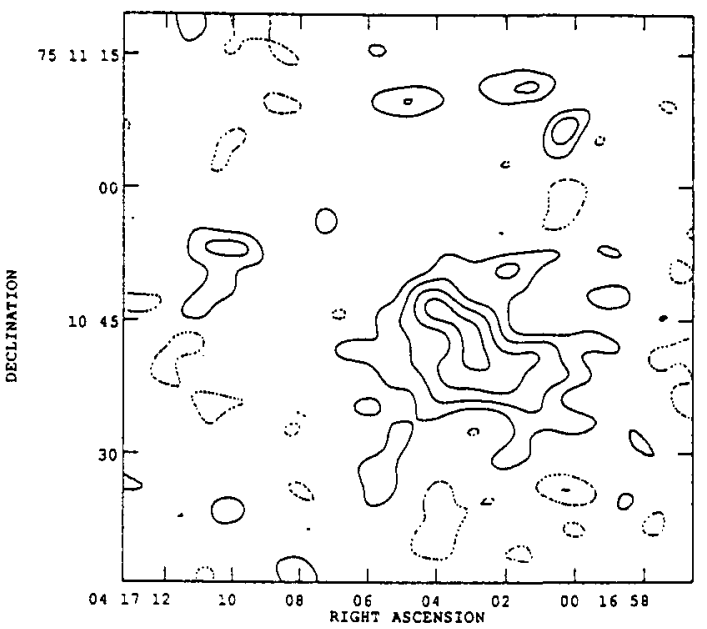

Fig.7. The CO (1-0) map of NGC 1530 (Ishizuki 1993). 
The $\mathrm{CO}(1-0)$ emission of the nuclear regions of ten galaxies have been observed (Ishizuki 1993). The high resolution images of three starburst galaxies indicate that the molecular gas at the small radii is associated with their circumnuclear starburst sites.

High resolution imagings for OMC-1 have been carried out with the molecular lines of $\mathrm{CS}(1-0), \mathrm{CS}(2-1)$, and $\mathrm{NH}_{3}$, and $\lambda \sim 3 \mathrm{~mm}$ continuum emissions (Murata 1990). Murata et al. (1993) have made multi-field mosaic images $\left(8^{\prime} \times 3^{\prime}\right)$, which reveal wiggled structures of the OMC ridge.

The survey project for protoplanetary disks has been made for 19 protostellar IRAS sources in Taurus (Fig. 8; Ohashi et al. 1992). There is a remarkable tendency that the detectability for the $98 \mathrm{GHz}$ continuum emission is small for protostar candidates (Ohashi et al. 1993). Kawabe et al. (1992) have discovered a rotating gas disk having a radius of about $500 \mathrm{AU}$ around GG Tau.

Shibata et al. (1993) have made aperture synthesis observations of $\mathrm{CO}(1-0)$ emission in three planetary nebulae, IRAS $21282+5050$, CRL 618, and M 1-7. In IRAS $21282+5050$, they found that CO gas forms an expanding toroid.

\section{REFERENCES}

Chikada, Y., et al. 1987, Proc. IEEE, 75, 1203

Ishiguro, M., et al. 1984, in Proc. Int. Symp. Millimeter and Submillimeter Radio Astronomy, Granada 78, reproduced in Instrumentation and Techniques for Radio Astronomy, P. Goldsmith (New York: IEEE Press), 151

Ishiguro, M., et al. 1990, in Submillimetre Astronomy, G. D. Watt and A. S. Webster (Dordrecht: Kluwer), 89

Ishizuki, S. 1993, Ph.D. thesis

Ishizuki, S., et al. 1993, this volume

Kawaguchi, N. 1992, in MWE'92 Microwave Workshop Digest, 27

Kawabe, R. et al. 1990, in the 3rd Asia-Pacific Microwave Conference Proceedings, Tokyo, 217

Kawabe, R., Sakamoto, K., Ishizuki, S., and Ishiguro 1992, ApJ, 397, L23

Kawabe, R., Ishiguro, M., Omodaka, T. Kitamura, Y., and Miyama, S. M. 1992, $A p J, 404, \mathrm{~L} 63$

Kawabe, R., Suziki, M., Morita, K.-I., Ohashi, M., and Ishiguro 1993, this volume

Morita, K.-I., et al. 1989, in Proc. the 1989 Int. Symp. Antennas and Propagations, Japan, 33

Morita, K.-I., Takahashi, T., Iawashita, H., and Kanzawa, T. 1991, in Proc. the Int. Conf. Accelerator and Large Experimental Physics Control Systems, Tsukuba, 214

Morita, K.-I. et al. 1992, PASJ, 44, 373

Murata, Y. 1990, Ph.D. thesis 
Murata, Y., Kawabe, R., Ishiguro, M., Morita, K.-I., Hasegawa, T., and Hayashi, M. 1993, this volume

Ohashi, N., Kawabe, R., Hayashi, M., and Ishiguro, M. 1992, in Proc. Planetary System: Formation, Evolution, and Detection, Pasadena, in press

Ohashi, N., Kawabe, R., Hayashi, M., and Ishiguro, M. 1993, this volume

Okumura, S. K., Kawabe, R., Ishiguro, M., Kasuga, T., Morita, K.-I., and Ishizuki, S. 1991, in Dynamics of Galaxies and their Molecular Distributions, ed. F. Combes (Dordrecht: Kluwer), 425

Sunada, K., Kawabe, R., and Inatani, J. 1993, this volume

Shibata, K., Deguchi, S., Kasuga, T., Tamura, S., Hirano, N., and Kameya, O. 1993 , this volume

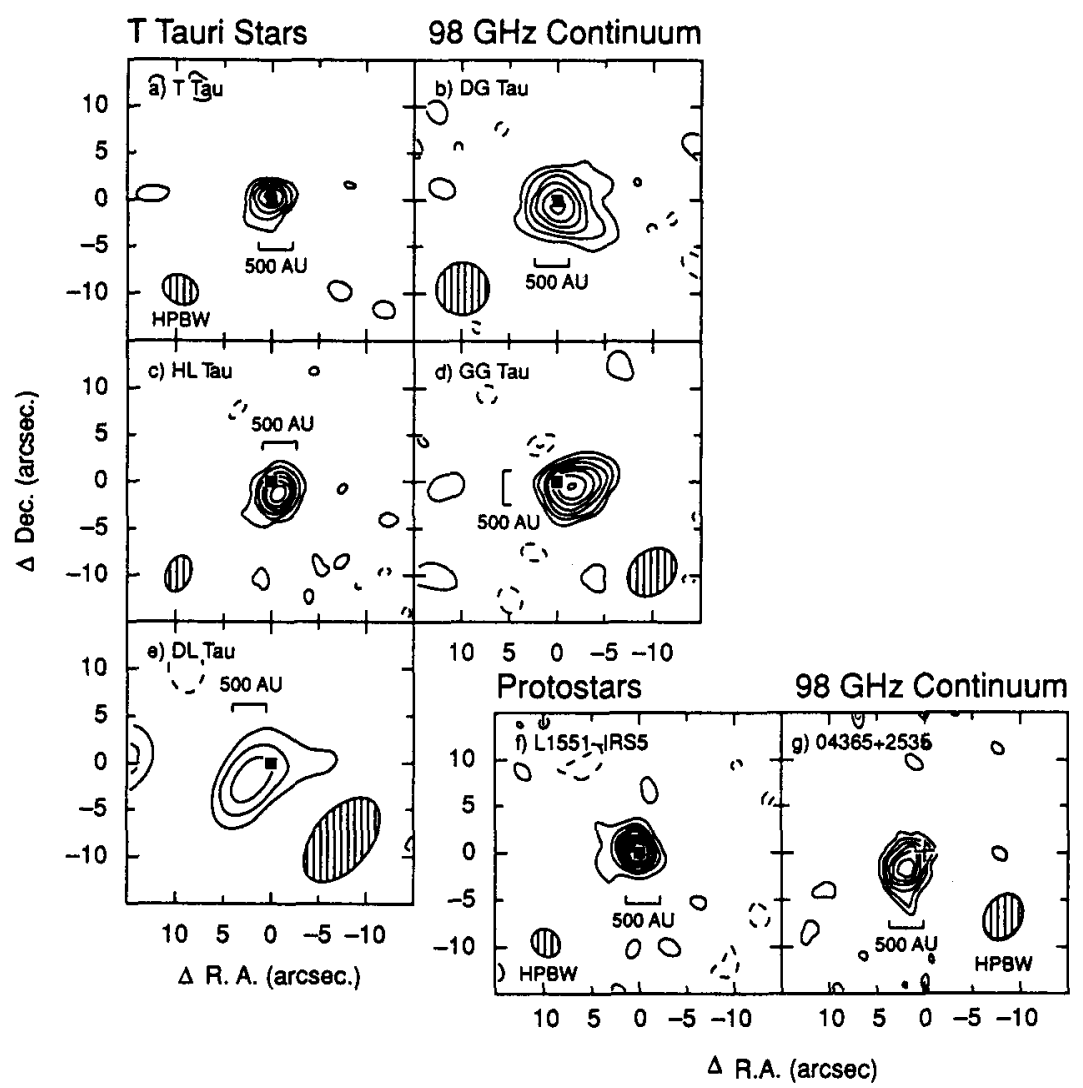

Fig.8. The $98 \mathrm{GHz}$ continuum maps of $\mathrm{T}$ Tauri stars and protostars Ohashi et al. (1992). 